\title{
SOME PROPERTIES OF CENTRAL RIGID RINGS
}

\author{
DUŠAN S. JOKANOVIĆ* \\ University of East Sarajevo \\ Faculty of Production and Management Trebinje \\ Stepe Stepanovića bb, 89 101, Bosnia and Herzegovina \\ *Corresponding author. E-mail: dusan.jokanovic@fpm.ues.rs.ba
}

\section{DOI : 10.20948/mathmontis-2020-48-1}

Summary. In this paper we deal with central rigid ring which is generalization of rigid ring. We consider how the notion of central rigidity can be transferred from the ring to the corresponding matrix and polynomial extension. Quasi-rigid rings will be also considered.

\section{INTRODUCTION}

In this article $\mathrm{R}$ denotes an associative ring with identity. Mehrabadi i Shakebi in [1] introduced a notion of central rigid ring. For endomorphism $\sigma$, a ring $\mathrm{R}$ is $\sigma$ central rigid if a $\sigma(a)=0$ implies $a \in C(R)$, where $\mathrm{C}(\mathrm{R})$ is center of ring $\mathrm{R}$. We know that a class of central rigid rings is generalization of class of rigid rings. An ideal $I$ of a ring $\mathrm{R}$ is called $\sigma$-ideal if $\sigma(I) \subseteq I$. We say that $\sigma$-ideal I is quasi $\sigma$-rigid if $a R \sigma(a) \subseteq I$ implies $a \in I$, for all $a \in R$ [2]. A ring $\mathrm{R}$ with an endomorphism $\sigma$ is quasi $\sigma$-rigid if ideal $\mathrm{I}=0$ is quasi $\sigma$-rigid which is equivalent to condition $a R \sigma(a)=0$ implies $a=0$. It is well known from [1] that the class of central rigid rings is closed for direct products, localizations, direct limits with injective maps, and isn't closed for homomorphic images. If $\sigma$ is endomorphism of a ring $\mathrm{R}$ then the map $\sigma$ can be naturally extended to an endomorphism $\sigma^{\prime}$ of the ring $R[x]$ by $\sigma^{\prime}\left(\sum_{i=0}^{n} a_{i} x^{i}\right)=$ $\sum_{i=0}^{n} \sigma\left(a_{i}\right) x^{i}$. In [1] is also shown that the notion of central rigidity can be transferred from ring $\mathrm{R}$ to corresponding ring of polynomials in linear variant. Let $\sigma$ be an endomorphism of $\mathrm{R}$. We use $R[x ; \sigma]$ to denote skew polynomial ring with the ordinary addition and the multiplication subject to the relation $x r=\sigma(r) x$ (see [3]).

\section{CENTRAL RIGIDITY VERSUS QUASI RIGIDITY IN POLYNOMIAL RINGS}

Recall that [4] the notion of quasi rigidity transferees from the ring $\mathrm{R}$ to the ring $\mathrm{R}[\mathrm{x}]$. If $\sigma$ is endomorphism of a ring $\mathrm{R}$ then the map $\sigma$ can be $\mathrm{n}$ extended to an endomorphism $\sigma^{\prime}$ of the ring $\mathrm{R}[\mathrm{x}]$ by equation

$$
\sigma^{\prime}\left(\sum_{i=0}^{n} a_{i} x^{i}\right)=\sum_{i=0}^{n} \sigma\left(a_{i}\right) x^{i}
$$

THEOREM 2.1. [3] If $\mathrm{R}$ is quasi $\sigma$-rigid then $\mathrm{R}[\mathrm{x}]$ is quasi $\sigma^{\prime}$-rigid ring.

PROOF. Let $f(x)=a_{0}+a_{1} x+\ldots+a_{n} x^{n}$ and $f(x) R[x] \sigma^{\prime}(f(x))=0$. Let

$$
f(x)=b_{0}+b_{1} x+\ldots+b_{m} x^{m},
$$

be an element from the ring $\mathrm{R}[\mathrm{x}]$. At first glance from the 


$$
\sum_{i=0}^{n} a_{i} x^{i} \sum_{j=0}^{m} b_{j} x^{j} \sum_{i=0}^{n} \sigma\left(a_{i}\right) x^{i}
$$

we obtain $a_{0} b_{0} \sigma\left(a_{0}\right)=0$ so that we have $a_{0}=0$.

$$
a_{0} b_{0} \sigma\left(a_{2}\right)+a_{0} b_{1} \sigma\left(a_{1}\right)+a_{0} b_{2} \sigma\left(a_{0}\right)+a_{1} b_{0} \sigma\left(a_{1}\right)+a_{2} b_{0} \sigma\left(a_{0}\right)+a_{2} b_{2} \sigma\left(a_{0}\right)=0
$$

so that we obtain $a_{1} b_{0} \sigma\left(a_{1}\right)=0$. Since $\mathrm{R}$ is quasi $\sigma$-rigid, we have $a_{1}=0$. Continuing in this way, since the coefficient of $x^{2 n+m-2}$ has to be zero and $a_{n-2}=0$ is obtained in the previous step, we have $a_{n-1} b_{m} \sigma\left(a_{n-1}\right)=0$. Using the quasi rigidity argument we have $a_{n-1}=0$. Finally at the end the coefficient of $x^{2 n+m}$ has to be zero, we obtain $a_{n} b_{m} \sigma\left(a_{n}\right)=0$, which means that $a_{n}=0$ and so $f(x)=0$.

From the fact that a class of quasi-rigid rings is generalization of class of rigid rings we obtain next result. We know from [4] that a class of quasi-rigid rings is closed for direct product constructions.

COROLLARY 2.1. If $\mathrm{R}$ is $\sigma$-rigid then $\mathrm{R}[\mathrm{x}]$ is $\sigma^{\prime}$-rigid ring.

Now we note a result from [5] about generating rigid rings with inner automorphism.

PROPOSITION 2.1. Let $\sigma$ be an inner automorphism of a reduced ring $\mathrm{R}$, then $\mathrm{R}$ is $\sigma$ rigid.

PROOF. Suppose that $r \sigma(r)=0, r \in R$. Since $\sigma$ is an inner automorphism there exists an invertible element $u \in R$, such that $r \sigma(r)=r u^{-1} r u=0$, so $r u^{-1} r=0$. This implies that $\left(r u^{-1}\right)^{2}=0$ and since $\mathrm{R}$ is reduced we get $r u^{-1}=0$, which means $r=u$. Then $\sigma(r)=r^{-1} r^{2}$, so $r \sigma(r)=r^{2}=0$, hence $r=0$. Therefore $\mathrm{R}$ is $\sigma$-rigid.

In the class of central rigid ring we have an extension of central rigidity notion only in a case when polynomial is linear. The ring $\mathrm{R}[\mathrm{x}]$ is called linear central $\sigma$-rigid if for any $f(x)=a_{0}+a_{1} x \in R[x], f(x) \sigma(f(x))=0$ implies that $f(x) \in C(R[x])$.

THEOREM 2.2 Let $\sigma$ be an endomorphism of a ring R. Then $\mathrm{R}$ is central $\sigma$-rigid if and only if $\mathrm{R}[\mathrm{x}]$ is linear central $\sigma$-rigid.

PROOF. Assume that $\mathrm{R}[\mathrm{x}]$ is linear central $\sigma$-rigid. Then $\mathrm{R}$ is central $\sigma$-rigid as a subring of $\mathrm{R}[\mathrm{x}]$. Conversely, assume that $\mathrm{R}$ is central $\sigma$-rigid and $f(x)=a_{0}+a_{1} x \in R[x]$ such that $f(x) \sigma(f(x))=0$. Then $a_{0} \sigma\left(a_{0}\right)=0$ and $a_{1} \sigma\left(a_{1}\right)=0$ and so $a_{0} ; a_{1} \in C(R)$, since $\mathrm{R}$ is central $\sigma$-rigid. Therefore, $f(x) \in C(R[x])$ and hence $\mathrm{R}[\mathrm{x}]$ is linear central Isigma-rigid.

Noting that the class of central rigid rings is closed for localization, and $R\left[x ; x^{-1}\right]=$ $R S^{-1}$, for $S=\left\{1, x, x^{2}, x^{3}, x^{4}, \ldots\right\}$, we recall next corollary from [1].

COROLLARY 2.2. Let $\mathrm{R}$ be a ring and $\sigma$ an automorphism of $\mathrm{R}$. Then the following are equivalent:

(1) $\mathrm{R}$ is central $\sigma$-rigid.

(2) $\mathrm{R}$ is linear central $\sigma$-rigid. 
(3) $R\left[x ; x^{-1}\right]$ is linear central $\sigma$-rigid.

\section{MATRIX CENTRAL RIGID RINGS}

In this section we give an example of matrix central rigid ring. At first glance for a ring $R$ we consider a following set of triangular matrices

$$
T_{n}(R)=\left\{\left[\begin{array}{lllll}
a_{11} & a_{12} & a_{13} & \ldots & a_{1 n} \\
0 & a_{22} & a_{23} & \ldots & a_{2 n} \\
0 & 0 & a_{33} & \ldots & a_{3 n} \\
\vdots & \vdots & \vdots & \ddots & \vdots \\
0 & 0 & 0 & \ldots & a_{n n}
\end{array}\right] \mid a_{i j} \in R\right\},
$$

and

$$
T(R, n)=R I_{n}+\sum_{i=1}^{n} \sum_{k=i+1}^{n} R E_{i j},
$$

where $E_{i j}$ is the matrix unit for all $i, j$ and $I_{n}$ is the identity matrix.

$$
T(R, n)=\left\{\left[\begin{array}{lllll}
a_{0} & a_{1} & a_{2} & \ldots & a_{n-1} \\
0 & a_{0} & a_{1} & \ldots & a_{n-2} \\
0 & 0 & a_{0} & \ldots & a_{3 n} \\
\vdots & \vdots & \vdots & \ddots & \vdots \\
0 & 0 & 0 & \ldots & a_{0}
\end{array}\right] \mid a_{i} \in R\right\},
$$

$T_{n}(R)$ and $T(R, n)$, as we know, are subrings of the triangular matrix rings with matrix addition and multiplication. Let $\alpha$ be endomorphism of ring $R$. It is well known that endomorphism $\alpha$ can be naturally extended to an endomorphism

$$
\bar{\alpha}: T_{n}(R) \rightarrow T_{n}(R)
$$

and

$$
\bar{\alpha}: T(R, n) \rightarrow T(R, n) .
$$

In the next part we use the notation from [3].

Let $E_{i j}=\left(e_{s t}: 1 \leq s, t \leq n\right)$ denotes $n \times n$ unit matrices over ring R, in which $e_{i j}=1$ and $e_{s t}=0$ when $s \neq i$ or $t \neq j, 0 \leq i, j \leq n$, for all $n \geq 2$. If $V=\sum_{i=1}^{n} E_{i, i+1}$, then

$$
V_{n}(R)=R I_{n}+R V+\ldots+R V^{n-1}
$$

is the subring of upper triangular skew matrices.In the next section we will show that under the assumption that $R[x] /\left(x^{n}\right)$ is central rigid we obtain that a ring $\mathrm{T}(\mathrm{R}, \mathrm{n})$ has the same property.

PROPOSITION 3.1. [1] Let $\alpha$ be an endomorphism of a ring $\mathrm{R}$. Let $\mathrm{S}$ be a ring and $\phi: R \rightarrow S$ an isomorphism. Then $\mathrm{R}$ is central $\alpha$-rigid if and only if $\mathrm{S}$ is central $\phi \alpha \phi^{-1}$-rigid ring.

THEOREM 3.1.Suppose that $\alpha$ is an endomorphism of ring $\mathrm{R}$. If the factor $\operatorname{ring} R[x] /$ $\left(x^{n}\right)$ is weak $\tilde{\alpha}$-central rigid, then $\mathrm{T}(\mathrm{R}, \mathrm{n})$ is $\tilde{\alpha}$-central rigid. 
PROOF. Suppose that $R[x] /\left(x^{n}\right)$ is $\tilde{\alpha}$-central rigid. In the next step we define the ring isomorphism $\theta: V_{n}(R) \rightarrow R[x] /\left(x^{n}\right)$ by

$$
\theta\left(r_{0} I_{n}+r_{1} V+. .+r_{n-1} V^{n-1}\right)=r_{0}+r_{1} x+. .+r_{n-1} x^{n-1}+\left(x^{n}\right) .
$$

We obtain that $V_{n}(R)$ is $\theta^{-1} \tilde{\alpha} \theta$-central rigid and calculate

$$
\begin{array}{r}
\theta^{-1} \tilde{\alpha} \theta \theta\left(r_{0} I_{n}+r_{1} V+. .+r_{n-1} V^{n-1}\right)=\theta^{-1} \tilde{\alpha}\left(r_{0}+r_{1} x+. .+r_{n-1} x^{n-1}+\left(x^{n}\right)\right)= \\
\quad=\theta^{-1}\left(\alpha\left(r_{0}\right)+\alpha\left(r_{1}\right) x+. .+\alpha\left(r_{n-1}\right) x^{n-1}+\left(x^{n}\right)\right)= \\
=\alpha\left(r_{0}\right) I_{n}+\alpha\left(r_{1}\right) V+\cdots+\alpha\left(r_{n-1}\right) V^{n-1}=\tilde{\alpha}\left(r_{0} I_{n}+r_{1} V+\cdots+r_{n-1} V^{n-1}\right) .
\end{array}
$$

which means that $V_{n}(R)$ is weak $\tilde{\alpha}$-central rigid [1]. In the next step we use isomorphism $f$ defined by isomorphism $f: R[x] /\left(x^{n}\right) \rightarrow T(R, n)$ given by

$$
f\left(a_{0}+a_{1} x+\ldots+a_{n-1} x^{n-1}\right)=\left(a_{0}, a_{1}, \ldots, a_{n-1}\right) .
$$

Now we use well known theorem of isomorphism of rings to obtain desired result.

We end this section with remark that a class of quasi rigid rings isn't closed for matrix extensions.

For a ring $\mathrm{R}$ and endomorphism $\sigma: R \rightarrow R$ we consider

$$
S_{4}=\left[\begin{array}{cccc}
a & a_{12} & a_{13} & a_{14} \\
0 & a & a_{23} & a_{24} \\
0 & 0 & a & a_{34} \\
0 & 0 & 0 & a
\end{array}\right], a, a_{i j} \in R,
$$

which is subring of $T_{4}(R)$. If $R$ is a $\sigma$ rigid and

$$
a=\left[\begin{array}{llll}
0 & 1 & 0 & 0 \\
0 & 0 & 0 & 0 \\
0 & 0 & 0 & 0 \\
0 & 0 & 0 & 0
\end{array}\right]
$$

easy calculation shows that $a S_{4} \bar{\sigma}(a)=0$, inspite the fact that $a \neq 0$, which shows that $S_{4}$ is not quasi $\sigma$-rigid (see also [6]).

\section{CONCLUSION}

This article deals with a class of rigid rings and its generalizations. Some of classes are closed for direct products, localizations as well as for direct limits. Main results are related to the possibility of extending property of rigidity from ring to some of its extensions. We proved that the property of central rigidity can be transferred from the ring to a corresponding matrix ring, under some conditions of factor ring. Further investigation on rigid rings can be found in recent work [6]. The results on skew rigid rings are extensively used in [7].

\section{REFERENCES}

[1] M. Mehrabadi and S. Sahebi, "Central $\sigma$-rigid rings", Palestine Journal of Mathematics, 6 (2), 569-572 (2017).

[2] T. Ozdin and M. Kosan, "Quasi $\sigma$-rigid rings", Int. J. Contemp. Math. Sciences, 3 (27), 13311335 (2011).

[3] D. Jokanović, "Properties of Armendariz rings and weak Armendariz rings", Publications de l'Institut Mathematique, 85, 131-137 (2009). 
[4] D. Jokanović, “A note to quasi rigid rings", Mathematica Montisnigri, 3, 14-17 (2008).

[5] H. Pourtaherian and I. Rakhimov, "On Hilbert Property of Rings", International Journal of Algebra, 5 (7), 301-308 (2011).

[6] C. Abdiôglu, S. Sahinkaya and A. Kör, "Rigid, quasi-rigid and matrix rings with $(\bar{\sigma}, 0)$ multiplication", Algebra and Discrete Mathematics, 17, 1-11 (2014).

[7] L. Ouyang, "Extensions of generalized $\alpha$-rigid rings", International Journal of Algebra, 3, 105$116(2008)$.

Received July 10, 2020 